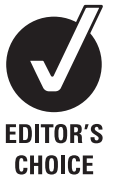

${ }^{1}$ Division of Interventional Cardiology, Royal Jubilee Hospital, Victoria, British Columbia, Canada

${ }^{2}$ King's College Hospital, London, UK

${ }^{3}$ University of Alberta, Alberta, Canada

${ }^{4}$ Division of Nephrology, University of British Columbia, Vancouver British Columbia, Canada

\section{Correspondence to} Dr Tycho Vuurmans, Victoria Heart Institute, 200-1900 Richmond Avenue, V8R 4R2 Victoria, British Columbia, Canada; tvuurmans@vhif.org

Accepted 4 June 2010 Published Online First 28 July 2010

\title{
Chronic kidney injury in patients after cardiac catheterisation or percutaneous coronary intervention: a comparison of radial and femoral approaches (from the British Columbia Cardiac and Renal Registries)
}

\author{
Tycho Vuurmans, ${ }^{1}$ Jonathan Byrne, ${ }^{2}$ Eric Fretz, ${ }^{1}$ Christian Janssen, ${ }^{3} \mathrm{~J}$ David Hilton, ${ }^{1}$
} W Peter Klinke, ${ }^{1}$ Ognjenka Djurdjev, ${ }^{4}$ Adeera Levin ${ }^{4}$

\begin{abstract}
Background Acute kidney injury (AKI) is a wellrecognised complication of cardiac catheterisation and percutaneous coronary intervention (PCI). However, the incidence of chronic kidney disease (CKD) after catheterisation and $\mathrm{PCl}$ has not been fully evaluated. A number of risk factors have been implicated in the development of AKI following cardiac catheterisation. Transradial access could lead to a lower incidence of CKD after catheterisation or PCI because of less catheter contact with aortic atheroma, and reduced potential for atheroembolism.

Objective To determine the incidence of CKD onset and its association with arterial access in patients after cardiac catheterisation or $\mathrm{PCl}$.

Methods and results Linkages between the British Columbia (BC) Cardiac Registry ( $\mathrm{N}=69$ 214) patients who underwent catheterisation or PCI between 1999 and 2005 and the BC Renal Database were determined. Within 6 months after the cardiac procedure $0.4 \%$ of patients developed dialysis dependency, $0.2 \%$ in the transradial versus $0.4 \%$ in the transfemoral group $(p<0.0001) ; 0.3 \%$ of patients developed stage 4 or $5 \mathrm{CKD}, 0.1 \%$ in the transradial versus $0.4 \%$ in the transfemoral group $(p<0.0001) ; 0.9 \%$ of patients developed new CKD, $0.2 \%$ in the transradial versus $1.2 \%$ in the transfemoral group $(p<0.0001)$. After adjusting for baseline characteristics the femoral access site had an OR of 4.36 (95\% Cl 2.48 to 7.66 ) for the development of the composite end point of new dialysis, new stage 4 or 5 CKD or new CKD.
\end{abstract}

Conclusions In this large database of current practice coronary catheterisation and $\mathrm{PCl}$, the incidence of CKD onset within 6 months of the procedure was $0.9 \%$. The transradial access site is associated with less CKD than the femoral approach.

\section{INTRODUCTION}

Acute kidney injury (AKI) is a well-recognised complication after cardiac catheterisation and percutaneous coronary intervention (PCI) and is associated with increased morbidity and mortality. ${ }^{1-6}$ AKI under these circumstances is thought to be due to the procedural use of radiographic contrast (contrast-induced AKI) and cholesterol emboli dislodged from the aorta by the diagnostic or guide catheter. ${ }^{7-9}$ The majority of
AKI after catheterisation or PCI is transient; however, a subset of patients require dialysis support. $^{3}{ }^{10-12}$ The number of patients who develop milder forms of chronic kidney disease (CKD) has not been described. The risk factors for AKI associated with contrast use and renal atheroemboli have been well studied, ${ }^{1-3} 713$ although intervention strategies remain controversial and varied.

The radial artery access is an alternative approach to performing cardiac catheterisation and PCI. It has advantages over the more traditional transfemoral approach owing to the easier compressibility of the radial artery and is associated with fewer vascular complications, particularly a reduction of access site bleeding. ${ }^{14-16}$ Furthermore, the radial approach may offer the additional advantage of avoiding passage through potential atheromatous aorta and renal vessels and may reduce the incidence of atheromata or emboli to the renal vascular beds. ${ }^{17}$ On the other hand, there have been reports of higher contrast use with the radial approach and concerns about possible subsequent renal effects from this more extensive dye load. ${ }^{18}$ Thus, uncertainty remains as to whether the radial access in comparison with the femoral access is associated with a lower incidence of renal failure after cardiac catheterisation or PCI. We undertook the following study to determine the incidence and risk factors, with specific attention to the arterial access site, for the development of CKD, as defined below, and more functionally than has been previously described after cardiac catheterisation and PCI.

\section{METHODS}

\section{Data linkage}

The British Columbia Cardiac Registry (BCCR) provides prospective demographic and procedural data for all patients who have undergone a catheterisation or PCI in British Columbia, Canada since 1994. This obligatory database ensures standardised data from all catheterisation centres is entered prospectively after each procedure into a central database maintained by a dedicated management team. For purposes of this study, data were used from all four centres that perform cardiac catheterisation and PCI. Demographic and clinical parameters were recorded from the BCCR, 

catheterisation or PCI, vascular access route, whether a second catheterisation or PCI took place in the next 6 months and if cardiac surgery (coronary artery bypass grafting and/or valve surgery) was performed in the 6 months after a catheterisation or PCI. Brachial access cases accounted for $0.1 \%$ of the entire cohort and were excluded.

Decisions to use the radial versus femoral approach were entirely operator dependent, and reflected 'real-world' practice.

Information about CKD was obtained by cross-referencing BCCR data with information from the BC Provincial Renal Agency database (Patient Outcome and Management Information System; PROMIS) so as to determine the incidence of new cases of $\mathrm{CKD}$, including dialysis and transplantation after coronary angiography. The PROMIS database is similarly obligatory, in that all patients in the province of British Columbia are registered into this provincial database after being referred to nephrologists and requiring care for CKD. Entry criteria for the database include estimated glomerular filtration rate $(\mathrm{eGFR})<60 \mathrm{ml} / \mathrm{min} / 1.73 \mathrm{~m}^{2}$ or diagnosis of kidney disease, presumed to be chronic on the basis of biopsy diagnosis, ultrasound or clinical history of deterioration and having been referred to a nephrologist. ${ }^{19}$ Patients who were undergoing dialysis at the time of cardiac catheterisation (baseline) were excluded.

eGFR was calculated using calibrated creatinine values and the abbreviated MDRD equation, as described previously. ${ }^{20}$

\section{Definition of new CKD status}

We defined new CKD status using a clinically based definition, and taking advantage of the PROMIS database. Thus, change in CKD status was considered present in patients who developed kidney disease requiring dialysis support, new stage 4 or 5 $\mathrm{CKD}^{21}$ defined as an eGFR of $15-29$ or $<15 \mathrm{ml} / \mathrm{min}$, respectively, or were newly referred to nephrologists, and deemed CKD by virtue of registration by the treating doctor, irrespective of eGFR. We defined a renal composite end point as belonging to either of these categories. These definitions for CKD had to be present within a window of 6 months after a cardiac catheterisation or PCI to be attributed to the event. The study was approved by the ethics committee of the University of British Columbia

\section{Protocols for prevention of AKI after catheterisation or PCI}

All participating hospitals used intravenous hydration with sodium chloride for prophylaxis in patients with renal failure at baseline $(e G F R<60 \mathrm{ml} / \mathrm{min}$ or creatinine $>130 \mu \mathrm{mol} / \mathrm{l})$ to varying degrees. The catheter laboratory directors reported infrequent use of $\mathrm{N}$-acetylcysteine or sodium bicarbonate before catheterisation or PCI during the study period of interest.

\section{Statistical analysis}

The Pearson $\chi^{2}$ test was used for comparison of categorical variables. The independent samples t test (with equal or unequal including clinical urgency, indication, risk factors, comorbidities,

variance determined by Levine's test), and analysis of variance were used for comparison of means of continuous variables. A $\mathrm{p}$ value of $<0.05$ was considered statistically significant for all analyses. Binary logistic regression was used to estimate the effect of baseline characteristics on occurrence of the combined renal end point, new dialysis, new stage 4 or 5 CKD (eGFR $<30 \mathrm{ml} / \mathrm{min}$ ), or new referral to nephrology within 6 months after catheterisation or PCI, with variables entered stepwise using the forward conditional likelihood ratio with $\mathrm{p}$-in $=0.05$ and $\mathrm{p}$-out $=0.10$. Propensity score matching was employed to compensate for differences in baseline conditions between patients undergoing a radial and femoral approach. The conditional probability (propensity score) of kidney function deterioration given baseline conditions (excluding access site) was estimated by binary logistic regression using a non-parsimonious model for referral to nephrology within 6 months after cardiac intervention based upon baseline clinical variables. The propensity score was used to match radial-access patients with femoral-access patients using stratification by deciles. All statistical calculations and analyses were performed using SPSS version 15 (SPSS, Chicago, Illinois, USA.)

\section{RESULTS}

Derivation of the cohort is described in figure 1. Note that the cohort of patients that underwent a cardiac procedure is vastly bigger than the eventual cohort of interest.

Baseline patient characteristics and clinical data are shown split by access site in table 1 .

Differences between the radial and femoral cohorts were statistically significant for most clinical variables, given the large sample size. Some of the differences noted may not be of clinical significance. The radial cohort was older, had more emergency procedures, a higher proportion of PCI and a higher percentage of patients underwent cardiac surgery within 6 months after catheterisation or PCI. The femoral cohort had a lower baseline eGFR, more patients with diabetes, congestive heart failure and cardiogenic shock. The radial access was associated with lower contrast use as compared with the femoral access for both catheterisation $(104.0 \pm 64.6$ vs $115.8 \pm 77.0 \mathrm{ml})$ and $\mathrm{PCI}$

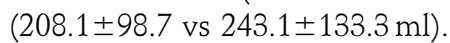

\section{Access site and incidence of dialysis, new stage 4 or 5 CKD or new referral to nephrologist}

Figure 2 shows the main outcome data, the incidence of dialysis, stage 4-5 CKD (eGFR $<30 \mathrm{ml} / \mathrm{min})$, newly diagnosed kidney disease and the composite renal end point within 6 months after cardiac catheterisation or PCI for the radial and femoral access respectively.

The incidence of new dialysis, new stage $4-5 \mathrm{CKD}$ and newly diagnosed kidney disease is low for the whole cohort $(0.4 \%, 0.3 \%$ and $0.9 \%$ ) and lower for the radial than for the femoral access site $(0.2 \%$ vs $0.4 \% ; p<0.0001,0.1 \%$ vs $0.3 \% ; p<0.0001,0.2 \%$ vs $1.1 \% ; \mathrm{p}<0.0001)$. The combined renal end point occurred at $0.5 \%$ for the radial cohort and $2.2 \%$ for the femoral cohort $(\mathrm{p}<0.0001)$.

Figure 1 Summary of procedures by access site and entry in BC Provincial Renal Agency database (Patient Outcome and Management Information System; PROMIS).

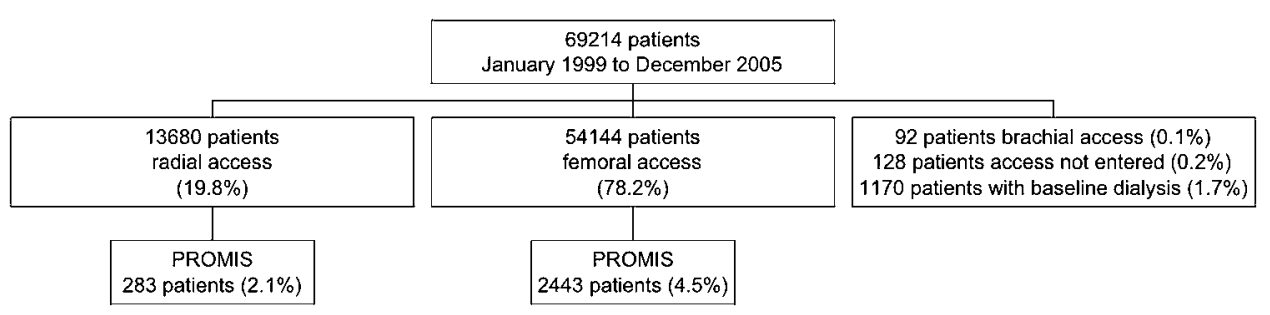


Table 1 Clinical variables in the whole cohort split by association with access site

\begin{tabular}{|c|c|c|c|}
\hline Variables & $\begin{array}{l}\text { Radial } \\
n=13680\end{array}$ & $\begin{array}{l}\text { Femoral } \\
n=54144\end{array}$ & p Value \\
\hline Age years (mean $\pm S D$ ) & $64.4 \pm 11.2$ & $63.9 \pm 12.0$ & $<0.0001$ \\
\hline Age $>75$ years & $17.5 \%$ & $18.2 \%$ & 0.072 \\
\hline BMI $\left(\mathrm{kg} / \mathrm{m}^{2}\right)$ & $28.4 \pm 5.5$ & $27.7 \pm 5.2$ & $<0.0001$ \\
\hline Male & $69.4 \%$ & $67.1 \%$ & $<0.0001$ \\
\hline Diabetes & $20.8 \%$ & $23.1 \%$ & $<0.0001$ \\
\hline Hyperlipidemia & $47.9 \%$ & $59.5 \%$ & $<0.0001$ \\
\hline Hypertension & $51.4 \%$ & $56.8 \%$ & $<0.0001$ \\
\hline Smoking current & $19.1 \%$ & $17.1 \%$ & $<0.0001$ \\
\hline Smoking quit & $41.9 \%$ & $46.0 \%$ & \\
\hline Congestive heart failure & $3.2 \%$ & $11.3 \%$ & $<0.0001$ \\
\hline$E F>50 \%$ & $82.3 \%$ & $66.7 \%$ & $<0.0001$ \\
\hline EF $30-50 \%$ & $13.8 \%$ & $27.2 \%$ & \\
\hline $\mathrm{EF}<30 \%$ & $3.9 \%$ & $6.0 \%$ & \\
\hline Cerebro vascular disease & $5.7 \%$ & $8.3 \%$ & $<0.0001$ \\
\hline Peripheral vascular disease & $8.9 \%$ & $8.8 \%$ & 0.70 \\
\hline eGFR (median) (ml/min) & 73.2 & 72.7 & $<0.0001$ \\
\hline eGFR <30 (ml/min) & $0.8 \%$ & $2.0 \%$ & $<0.0001$ \\
\hline eGFR30-60 (ml/min) & $22.2 \%$ & $23.4 \%$ & \\
\hline eGFR $>60(\mathrm{ml} / \mathrm{min})$ & $77.0 \%$ & $74.6 \%$ & \\
\hline ACS & $48.6 \%$ & $53.7 \%$ & $<0.0001$ \\
\hline Catheterization only & $68.3 \%$ & $81.3 \%$ & $<0.0001$ \\
\hline $\mathrm{PCl}$ & $31.7 \%$ & $18.7 \%$ & \\
\hline Elective procedure & $43.5 \%$ & $43.2 \%$ & 0.004 \\
\hline Urgent procedure & $48.8 \%$ & $49.8 \%$ & \\
\hline Emergent procedure & $7.7 \%$ & $7.0 \%$ & \\
\hline Contrast amount ml (cath) & $104.0 \pm 64.6$ & $115.8 \pm 77.0$ & $<0.0001$ \\
\hline Contrast amount ml (PCI) & $208.1 \pm 98.7$ & $243.1 \pm 133.3$ & $<0.0001$ \\
\hline Non-Ionic contrast & $53.7 \%$ & $76.9 \%$ & $<0.0001$ \\
\hline Low Osmolar contrast & $0.7 \%$ & $4.0 \%$ & \\
\hline lonic contrast & $37.0 \%$ & $11.4 \%$ & \\
\hline Low lonic contrast & $0.2 \%$ & $0.3 \%$ & \\
\hline Cardiogenic shock & $0.2 \%$ & $0.5 \%$ & 0.001 \\
\hline $\begin{array}{l}\text { Cardiac surgery }<6 \text { months after cardiac } \\
\text { event }\end{array}$ & $21.3 \%$ & $20.3 \%$ & 0.009 \\
\hline Second event $<6$ months & $23.2 \%$ & $22.5 \%$ & 0.066 \\
\hline
\end{tabular}

$\mathrm{BMI}=$ Body Mass Index; $\mathrm{EF}=$ Ejection fraction; eGFR=estimated glomerular filtration rate $\mathrm{ACS}=$ Acute coronary syndrome; $\mathrm{PCl}=$ Percutaneous coronary intervention.

\section{Logistic regression and associations of new CKD}

Baseline characteristics from table 1 were used in binary logistic regression for the composite renal outcome. The results are shown in table 2.

The resulting independent predictors were age, emergency procedure, diabetes, hypertension, cerebrovascular disease, congestive heart failure, ionic contrast use, cardiac surgery $<6$ months after cardiac event, a second event $<6$ months and access site. The femoral access site was associated with a more than fourfold increased risk for the composite renal end point across the entire cohort. When patients with an eGFR $<30 \mathrm{ml} /$ min at baseline were excluded from the analysis, the femoral access site had an OR of 2.6 (95\% CI 1.82 to 3.79) for the development of the composite renal end point.

\section{Propensity analysis}

Three different matched sets were created to conform to the groups in figure 2: patients who developed kidney disease requiring dialysis support, new stage 4 or $5 \mathrm{CKD}$ defined as an eGFR of $15-29$ or $<15 \mathrm{ml} / \mathrm{min}$, respectively, or patients who were newly referred to nephrologists. The incidence of new dialysis in the matched groups of 12980 radial and 12980 femoral-access patients was 17 in the former $(0.1 \%)$ and 40 in the latter $(0.3 \%),(p=0.002)$. The incidence of new stage 4 or 5

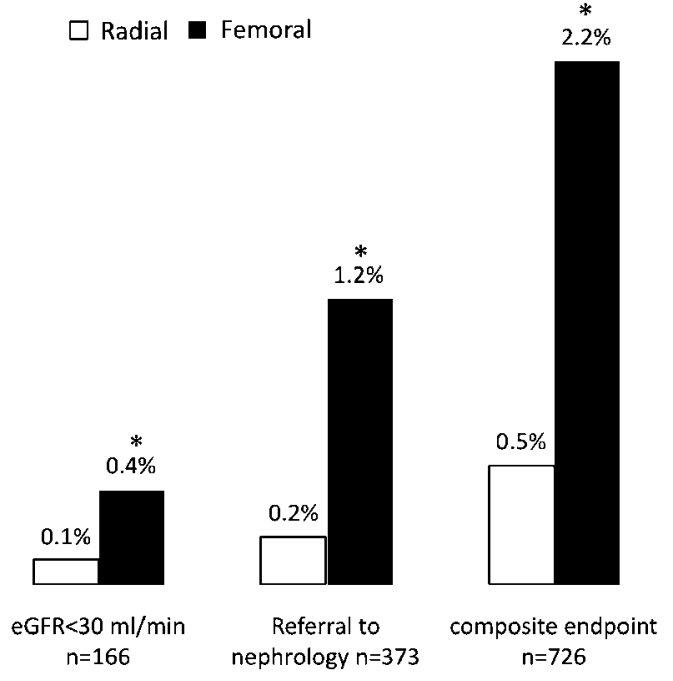

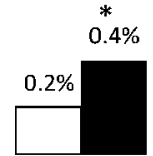

Dialysis $n=254$

Figure 2 Comparison of the radial and femoral access site for $\mathrm{PCl}$ or cardiac catheterisation on the outcomes dialysis $<6$ months for all patients, estimated glomerular filtration rate (eGFR) $<30 \mathrm{ml} / \mathrm{min}$ $<6$ months for patients with baseline eGFR $>30 \mathrm{ml} / \mathrm{min}$, new referral to nephrology $<6$ months for patients with eGFR $>60 \mathrm{ml} / \mathrm{min}$ at baseline and composite end point for adverse renal events. ${ }^{*} p<0.0001$.

CKD in the matched groups of 10670 radial and 10670 femoral patients was 15 in the former $(0.1 \%)$ and 39 in the latter $(0.4 \%)$, $(\mathrm{p}=0.001)$. New referral to nephrology in the matched groups of 8261 radial and 8261 femoral patients was 15 in the former $(0.2 \%)$ and 89 in the latter $(1.1 \%),(p=0.0001)$.

\section{DISCUSSION}

In this large cohort analysis of contemporary practice coronary catheterisation and PCI, the incidence of CKD onset within 6 months of the procedure is $0.9 \%$. The transradial access site is associated with less CKD than the femoral approach.

AKI is not uncommon after cardiac catheterisation and PCI and is usually transient. A small proportion of patients continue on chronic dialysis. ${ }^{10}{ }^{11}$ If dialysis is needed after PCI it is associated with a highly increased mortality rate. ${ }^{10} 11$ In our study we found that the incidence of dialysis within 6 months after catheterization or PCI was $0.4 \%$, which is comparable to the $0.4 \%$ incidence of dialysis found by Freeman et a $l^{10}$ and $0.7 \%$ found by Gruberg et al. ${ }^{12}$ Unlike previous studies, which focused on AKI or the need for dialysis, we report here the incidence of new CKD stage $4-5$ (eGFR $<30 \mathrm{ml} / \mathrm{min})$ in $0.3 \%$ of patients with an eGFR $>30 \mathrm{ml} / \mathrm{min}$ at baseline and newly diagnosed kidney disease within 6 months after a catheterisation or PCI in

Table 2 Multivariate predictors of composite end point for adverse renal events using binary logistic regression

\begin{tabular}{llc}
\hline & OR (95\% Cl) & p value \\
\hline Age & $1.02(1.01$ to 1.03$)$ & 0.001 \\
Emergency & $2.05(1.38$ to 3.06$)$ & $<0.0001$ \\
Urgency & $0.57(0.46$ to 0.71$)$ & $<0.0001$ \\
Diabetes & $1.44(1.15$ to 1.81$)$ & 0.002 \\
Hypertension & $1.27(1.02$ to 1.59$)$ & $<0.0001$ \\
Cerebrovascular disease & $1.53(1.08$ to 2.16$)$ & 0.016 \\
Congestive heart failure & $2.38(1.80$ to 3.15$)$ & $<0.0001$ \\
lonic contrast use & $0.24(0.12$ to 0.48$)$ & $<0.0001$ \\
Cardiac surgery $<6$ months after cardiac event & $4.42(3.57$ to 5.48$)$ & $<0.0001$ \\
Second event $<6$ months & $1.46(1.11$ to 1.92$)$ & 0.006 \\
Femoral access & $4.36(2.48$ to 7.66$)$ & $<0.0001$ \\
\hline
\end{tabular}


$0.9 \%$ of patients with an eGFR $>60 \mathrm{ml} / \mathrm{min}$ at baseline. These percentages are higher than the estimated annual incidence of $\mathrm{CKD}$ at $0.17 \%$ that was found in a British general population cohort over a 5.5 -year period of follow-up. ${ }^{22}$

In our study the transradial access site is associated with less CKD than the femoral approach after adjusting for baseline characteristics. These differences persisted in large propensity-matched cohorts.

\section{Limitations and strengths}

The data are non-randomised and therefore serve to generate hypotheses rather than proving that the radial approach may have fewer long-term complications. By adjusting for all known recorded variables we have controlled for differences in risk between the femoral and radial cohorts, which might otherwise account for the observations. Nonetheless, unknown confounders of risk difference between the radial and femoral cohorts may persist. There was a difference in radial access use between the different PCI centres. One of the centres performed a majority of cases through the radial access approach (71.1\%), whereas the other centres used radial access only in a minority of cases $(4.4 \%$, $3.0 \%$ and $1.3 \%$, respectively). This can potentially introduce bias through a 'centre effect'. However, in examining the various risk factor profiles, these patients appeared sicker, which would conservatively bias the difference estimates against the radial access site.

Explaining the potential contributors to the outcomes requires more detailed review. A number of differences are determined

1. Contrast use was lower in the radial group than in the femoral group. This is the first time such a difference has been found in a large study cohort. Earlier, smaller, studies have found both higher ${ }^{18}$ and equal ${ }^{23} 24$ usage of contrast with the radial access, but this is the first study to describe a consistently lower use of contrast in transradial procedures. This may be explained, at least partly, as due to increased overall experience with radial access catheterisation and PCI. In this study the amount of contrast was not an independent predictor for renal outcomes; however, earlier studies have found an important association between contrast volume use and incidence of AKI. ${ }^{2} 1011132526$

2. Cholesterol embolisation is a well-recognised, although rare, complication after cardiac catheterisation that has been implicated in the development of renal dysfunction in $\sim 0.9 \%$ of patients. ${ }^{7}$ The abdominal aorta is one of the areas most heavily affected with atherosclerotic plaque, and aortic 'scraping' has been documented with coronary catheter advancement ${ }^{2} 27$ through the femoral access. This can disrupt plaque material and induce renal cholesterol emboli. ${ }^{17}$ A recent study found that most patients with an iatrogenic form of atheroembolic renal disease had cardiac catheterisation via the femoral artery as the most common precipitating factor. ${ }^{9}$ When the catheter is advanced through the radial access it has less contact with aortic atheroma and, potentially, a reduced likelihood of renal atheroembolisation. However, since we do not have data on cholesterol emboli in both groups, this explanation remains speculative.

3. A third possibility is that the radial access leads to less CKD because it reduces access site bleeding ${ }^{16}$ and reduces the need for blood transfusion after PCI as compared with the femoral access. ${ }^{28}$ Bleeding has been shown to be associated with AKI after $\mathrm{PCI}^{2}$ and low haematocrit before $\mathrm{PCI}$ is a risk factor for contrast-induced $\mathrm{AKI}^{29} \mathrm{~A}$ recent study also found blood transfusion to be an independent predictor of post-PCI nephropathy. ${ }^{30}$ In a recent cardiac surgical study perioperative anaemia and blood transfusion were independently associated with the development of AKI. ${ }^{31}$ Even though we do not have data on bleeding and blood transfusion in our study, this is a potential explanation for at least some of the observed difference in renal outcomes between the radial and femoral approach. The other independent predictors that were found for the combined renal end point were age, emergency procedure, diabetes, hypertension, cerebrovascular disease, congestive heart failure, cardiac surgery within 6 months after the catheterisation or PCI and a second procedure within 6 months. Age, emergency procedure, diabetes, hypertension, cerebrovascular disease, congestive heart failure and cardiac surgery have also been described as independent risk factors for $\mathrm{AKI}^{2}{ }^{4} 13 \quad 25 \quad 31$ and dialysis ${ }^{10}{ }^{12}$ by others. A second procedure within 6 months after the original procedure can be expected to be a predictor of CKD because of the additional associated risk of a new catheterisation or PCI. Many of these risk factors were present in the radial group.

The data we present are observational, and non-randomised, albeit prospectively collected in real time. Thus, the limitations of this study design are clear. We have depended on statistical methods to adjust for differences between the radial and femoral cohorts, which were statistically, though possibly not clinically, significant for most clinical variables. Of course, we were not able to control for residual confounding of variables not readily available in the database, nor adjust for potential differences in classification/ categorisation of variables in different clinical centres.

Although a strength of this study is the very large number of patients and procedures, even minor differences in demographics become highly statistically significant (table 1) and thus caution should be used in reviewing some of the comparisons.

\section{CONCLUSIONS}

In this large database of current practice coronary catheterisation and PCI, the incidence of CKD onset within 6 months of the procedure is $0.9 \%$, and the transradial access site appears to be associated with less new CKD than the femoral approach. Since this conclusion is based on observational and non-randomised data, this study is primarily hypothesis generating. This observation needs confirmation by other large registries and randomised prospective trials, as the implications for high-risk populations regarding PCI related CKD may be profound.

Acknowledgements We acknowledge the contributions of Karin Humphries and staff at the BC Cardiac Registry and BC Provincial Renal Agency database for help with data collection.

Funding Victoria Heart Institute.

Competing interests None

Ethics approval This study was conducted with the approval of the University of British Columbia.

Provenance and peer review Not commissioned; externally peer reviewed.

\section{REFERENCES}

1. Brown JR, DeVries JT, Piper WD, et al. Serious renal dysfunction after percutaneous coronary interventions can be predicted. Am Heart J 2008;155:260-6.

2. Dangas G, lakovou I, Nikolsky E, et al. Contrast-induced nephropathy after percutaneous coronary interventions in relation to chronic kidney disease and hemodynamic variables. Am J Cardiol 2005;95:13-19.

3. McCullough PA, Wolyn R, Rocher LL, et al. Acute renal failure after coronary intervention: incidence, risk factors, and relationship to mortality. Am J Med 1997; 103:368-75.

4. Lindsay J, Apple S, Pinnow EE, et al. Percutaneous coronary intervention-associated nephropathy foreshadows increased risk of late adverse events in patients with normal baseline serum creatinine. Catheter Cardiovasc Interv 2003;59:338-43. 
5. Gruberg L, Mintz GS, Mehran R, et al. The prognostic implications of further renal function deterioration within $48 \mathrm{~h}$ of interventional coronary procedures in patients with pre-existent chronic renal insufficiency. J Am Coll Cardiol 2000:36:1542-8.

6. Rihal CS, Textor SC, Grill DE, et al. Incidence and prognostic importance of acute renal failure after percutaneous coronary intervention. Circulation 2002:105:2259-64.

7. Fukumoto $\mathbf{Y}$, Tsutsui $H$, Tsuchihashi $M$, et al. The incidence and risk factors of cholesterol embolization syndrome, a complication of cardiac catheterization: a prospective study. J Am Coll Cardiol 2003;42:211-16.

8. Drost H, Buis B, Haan D, et al. Cholesterol embolism as a complication of left heart catheterisation. Report of seven cases. Br Heart J 1984:52:339-42.

9. Scolari F, Ravani P, Gaggi $R$, et al. The challenge of diagnosing atheroembolic renal disease: clinical features and prognostic factors. Circulation 2007;116:298-304.

10. Freeman RV, O'Donnell M, Share D, et al. Nephropathy requiring dialysis after percutaneous coronary intervention and the critical role of an adjusted contrast dose. Am J Cardiol 2002;90:1068-73.

11. Nikolsky E, Mehran R, Turcot D, et al. Impact of chronic kidney disease on prognosis of patients with diabetes mellitus treated with percutaneous coronary intervention. Am J Cardiol 2004:94:300-5.

12. Gruberg L, Mehran R, Dangas G, et al. Acute renal failure requiring dialysis after percutaneous coronary interventions. Catheter Cardiovasc Interv 2001;52:409-16.

13. Mehran R, Aymong ED, Nikolsky E, et al. A simple risk score for prediction of contrast-induced nephropathy after percutaneous coronary intervention: development and initial validation. J Am Coll Cardiol 2004:44:1393-9.

14. Kiemeneij F, Laarman GJ, Odekerken D, et al. A randomized comparison of percutaneous transluminal coronary angioplasty by the radial, brachial and femoral approaches: the access study. J Am Coll Cardiol 1997:29:1269-75.

15. Agostoni P, Biondi-Zoccai GG, de Benedictis ML, et al. Radial versus femoral approach for percutaneous coronary diagnostic and interventional procedures; Systematic overview and meta-analysis of randomized trials. J Am Coll Cardiol 2004:44:349-56

16. Pristipino C, Pelliccia F, Granatelli A, et al. Comparison of access-related bleeding complications in women versus men undergoing percutaneous coronary catheterization using the radial versus femoral artery. Am J Cardiol 2007:99:1216-21.

17. Karalis DG, Quinn V, Victor MF, et al. Risk of catheter-related emboli in patients with atherosclerotic debris in the thoracic aorta. Am Heart J 1996;131:1149-55.
18. Jaffe R, Hong T, Sharieff W, et al. Comparison of radial versus femoral approach for percutaneous coronary interventions in octogenarians. Catheter Cardiovasc Interv 2007:69:815-20

19. Levin A, Djurdjev 0, Duncan J, et al. Haemoglobin at time of referral prior to dialysis predicts survival: an association of haemoglobin with long-term outcomes. Nephrol Dial Transplant 2006:21:370-7.

20. Komenda P, Beaulieu M, Seccombe D, et al. Regional implementation of creatinine measurement standardization. J Am Soc Nephrol 2008;19:164-9.

21. Inrig JK, Patel UD, Briley LP, et al. Mortality, kidney disease and cardiac procedures following acute coronary syndrome. Nephrol Dial Transplant 2008;23:934-40.

22. Drey N, Roderick $P$, Mullee $M$, et al. A population-based study of the incidence and outcomes of diagnosed chronic kidney disease. Am J Kidney Dis 2003:42:677-84.

23. Pristipino C, Trani C, Nazzaro MS, et al. Major improvement of percutaneous cardiovascular procedure outcomes with radial artery catheterisation: results from the PREVAIL study. Heart 2009;95:476-82.

24. Cantor WJ, Puley G, Natarajan MK, et al. Radial versus femoral access for emergen percutaneous coronary intervention with adjunct glycoprotein Ilb/llla inhibition in acute myocardial infarction-the RADIAL-AMI pilot randomized trial. Am Heart $J$ 2005; 150:543-9

25. Bartholomew BA, Harjai KJ, Dukkipati S, et al. Impact of nephropathy after percutaneous coronary intervention and a method for risk stratification. Am J Cardiol 2004;93:1515-19.

26. Laskey WK, Jenkins C, Selzer F, et al. Volume-to-creatinine clearance ratio: a pharmacokinetically based risk factor for prediction of early creatinine increase afte percutaneous coronary intervention. J Am Coll Cardiol 2007;50:584-90.

27. Keeley EC, Grines CL. Scraping of aortic debris by coronary guiding catheters: a prospective evaluation of 1,000 cases. J Am Coll Cardiol 1998;32:1861-5.

28. Chase AJ, Fretz EB, Warburton WP et al. Association of the arterial access site a angioplasty with transfusion and mortality: the M.O.R.T.A.L study (Mortality benefit of reduced transfusion after percutaneous coronary intervention via the Arm or Leg) Heart 2008:94:1019-25.

29. Nikolsky E, Mehran R, Lasic Z, et al. Low hematocrit predicts contrast-induced nephropathy after percutaneous coronary interventions. Kidney Int 2005;67:706-13.

30. Roy P, Raya V, Okabe T, et al. Incidence, predictors, and outcomes of postpercutaneous coronary intervention nephropathy in patients with diabetes mellitus and normal baseline serum creatinine levels. Am J Cardiol 2008:101:1544-9.

31. Karkouti K, Wijeysundera DN, Yau TM, et al. Acute kidney injury after cardiac surgery: focus on modifiable risk factors. Circulation 2009;119:495-502. 